\title{
Development and validation of a risk scoring model for postoperative adult moyamoya disease
}

\author{
*Moinay Kim, MD, ${ }^{1}$ Wonhyoung Park, MD, PhD, ${ }^{1}$ Yeongu Chung, MD, ${ }^{2}$ Si Un Lee, MD, ${ }^{3}$ \\ Jung Cheol Park, MD, PhD, ${ }^{1}$ Do Hoon Kwon, MD, PhD, ${ }^{1}$ Jae Sung Ahn, MD, PhD, ${ }^{1}$ and \\ Seungjoo Lee, MD, PhD'
}

\begin{abstract}
1Department of Neurosurgery, Asan Medical Center, University of Ulsan College of Medicine, Seoul; ${ }^{2}$ Department of Neurosurgery, Kangbuk Samsung Medical Center Hospital, Seoul; and ${ }^{3}$ Department of Neurosurgery, Seoul National University Bundang Hospital, Seongnam, Republic of Korea
\end{abstract}

\begin{abstract}
OBJECTIVE The current grading system for moyamoya disease (MMD) is focused on angiographic studies with limited clinical application. The authors aimed to determine relevant factors that may impact postoperative outcome and establish a scoring system to predict the functional outcome.
\end{abstract}

METHODS Adult patients with MMD who underwent treatment between 1998 and 2016 were included. Factors such as age, sex, comorbidity, smoking, MMD family history, initial presentation, multimodal imaging modalities, and types of surgical revascularization were thoroughly reviewed. These factors were analyzed to determine possible risk factors related to unfavorable 6-month postoperative outcomes using the modified Rankin Scale (mRS) (unfavorable: mRS score $\geq 3$ ). A scoring system was developed using these independent risk factors to predict the outcome and validated using prospectively collected data from multiple centers between 2017 and 2018.

RESULTS Of 302 patients for whom applications were submitted, 260 patients ( 321 hemispheres) met the diagnostic criteria. In multivariate analysis, hyperlipidemia, smoking, cerebral infarction on preoperative CT or MRI, and moderately to severely reduced regional cerebrovascular reserve results from Diamox SPECT were significantly related to unfavorable outcome. The authors developed a scoring system and stratified patients into risk groups according to their scores: low-risk (score 0-3), intermediate-risk (score 4-6), and high-risk (score 7-9) groups. This model demonstrated both good discrimination and calibration using C-statistics and the Hosmer-Lemeshow goodness-of-fit test showing 0.812 $(95 \% \mathrm{Cl} 0.743-0.881)(p=0.568)$ for the development and $0.954(95 \% \mathrm{Cl} 0.896-1)(p=0.097)$ for the temporal and external validation cohort.

CONCLUSIONS The authors' scoring system is readily adoptable to predict the postoperative outcome for MMD. Their data revealed the importance of smoking and hyperlipidemia, which were the only modifiable factors included in the scoring system. The authors validated their scoring system both internally and externally and maintained good performance, highlighting the system's generalizability and reliability.

https://thejns.org/doi/abs/10.3171/2020.2.JNS193221

KEYWORDS adult; cerebrovascular; moyamoya disease; prognosis; scoring; stroke; surgery; vascular disorders

$\mathrm{M}$ OYAMOYA disease (MMD) is characterized by idiopathic, chronic arteriopathy of the internal carotid arteries (ICAs) and their proximal vessels, which may lead to symptoms of cerebral ischemia or hemorrhage. ${ }^{1}$ The pathophysiology of MMD has not been prospectively defined, and the cause is not well known. In 1969, Suzuki and Takaku developed an MMD classification system using cerebral angiography. ${ }^{2}$ This classification, known as Suzuki staging, is currently the most widely adopted system for assessing the degree of stenosis, occlusion, and revascularization of MMD. Despite its popularity, Suzuki staging is solely an anatomy-dependent criterion because the stage is determined only on the basis of cerebral angiography findings, which do not sufficiently demonstrate hemodynamic compromise and hemodynamic vascular reserve status. ${ }^{3}$ Hence, Suzuki staging is limited in predicting neurological outcomes and related surgical risk.

ABBREVIATIONS EDAMS = encephaloduroarteriomyosynangiosis; EDAS = encephaloduroarteriosynangiosis; GEE = generalized estimating equation; ICA = internal carotid artery; MMD = moyamoya disease; $m R S$ = modified Rankin Scale; $P C A=$ posterior cerebral artery; rCVR = regional cerebrovascular reserve; ROC = receiver operating characteristic; TFCA = transfemoral catheter angiography.

SUBMITTED November 28, 2019. ACCEPTED February 26, 2020.

INCLUDE WHEN CITING Published online May 8, 2020; DOI: 10.3171/2020.2.JNS193221.

* M.K. and W.P. contributed equally to this work, and J.S.A. and S.L. contributed equally to this work. 
The incidence of postoperative ischemic adverse events in MMD is approximately $3 \%-7.7 \%,{ }^{4}$ which is not low. However, the association between risk factors and postoperative outcome of MMD is not well understood. In addition, the outcome of MMD is difficult to predict, and choosing an improper treatment plan can further worsen the outcome. Previous studies focusing on prognosis of MMD were largely dependent on angiographic studies..$^{5-9}$ However, these studies are not well suited for a prediction model because they were usually small study series and had methodological weaknesses such as lack of a large validation cohort. Thus, these scoring systems have less potential and value for application in clinical practice. The severity of arteriopathy and its association with vascular morphology, cerebrovascular hemodynamics, and clinical symptoms are essential prerequisites for developing a management plan. However, this may be limited by a single angiographic study alone. Patient clinical history as well as biomarkers and radiological studies should be considered in combination to precisely predict the outcome.

The objective of our study was to systematically identify the risk factors of MMD and to develop a scoring system that involves distinct clinical features of MMD that is also simple, precise, and readily adoptable at most institutions. Furthermore, we aimed to validate our scoring system via prospectively collected multicenter patients to examine its generalizability and reliability.

\section{Methods \\ Patient Population}

Appropriate institutional review board approval and patient consent were obtained for this study.

\section{Development Cohort}

The development cohort was retrospectively collected from a tertiary medical center, Asan Medical Center, in Seoul, Korea. Records from MMD patients admitted to our institution between January 1998 and December 2016 were thoroughly reviewed. The criteria for inclusion of patients in our study were 1) diagnosis of MMD by transfemoral catheter angiography (TFCA) in agreement with Japanese MMD guideline ${ }^{10}$ and their revision by the 2015 Research Committee for MMD of the Japanese Ministry of Health, Labour and Welfare; 2) age $\geq 18$ years; 3) history of surgical revascularization (direct, indirect, or combined bypass); and 4) postoperative 6-month modified Rankin Scale (mRS) score. Exclusion criteria included moyamoya syndrome that is a secondary moyamoya phenomenon caused by Down syndrome, neurofibromatosis type 1 , hyperthyroidism, autoimmune disease, head trauma, or head and neck irradiation. ${ }^{1,11}$ Patients who had previously undergone unilateral surgical revascularization were also included if the disease encroached on the contralateral hemisphere.

\section{Validation Cohorts}

We included the following 3 different validation cohorts in our study to verify the compatibility and efficiency of our scoring system: internal, temporal, and external. Patients in the internal validation cohort underwent revascu- larization surgery at the same time and in the same institution as those in the development cohort, between January 1998 and December 2016. Temporal validation samples were prospectively collected from the same institution as the development cohort (Asan Medical Center) but at a later time point, between January 2017 and October 2018. External validation included a prospective cohort from 2 different academic institutions in which revascularization surgery was performed between January 2017 and October 2018 .

\section{Data Collection and Definitions}

The related data were obtained from the medical records by a physician and trained research nurse. In this study, the following candidate factors were analyzed: 1) sex; 2) comorbidities, including hypertension (previous hypertension diagnosis or taking related medication), diabetes mellitus (previous diabetes mellitus diagnosis or taking related medication), hyperlipidemia (total cholesterol $\geq 240 \mathrm{mg} / \mathrm{dL}$, triglyceride $\geq 200 \mathrm{mg} / \mathrm{dL}$, high-density lipoprotein cholesterol $\leq 40 \mathrm{mg} / \mathrm{dL}$, low-density lipoprotein cholesterol $\geq 150 \mathrm{mg} / \mathrm{dL}$, or lipid-lowering medication use), ischemic heart disease (previous diagnosis of angina or myocardial infarction or related medication in use), and cigarette smoking (smoking more than 1 cigarette per day within 1 year prior to the operation); 3) family history of MMD; 4) number of surgical revascularizations; 5) initial presenting symptoms of headache, dizziness, dysarthria, extremity weakness or paresthesia, seizure or loss of consciousness, or visual field deficit; 6) initial CT and MRI findings of symptomatic cerebral infarction or hemorrhage; 7) Suzuki stage according to TFCA findings; 8) posterior cerebral artery (PCA) involvement; 9) preoperative SPECT results of regional cerebrovascular reserve (rCVR): mildly to moderately reduced, moderately reduced, and moderately to severely reduced; 10) types of surgical revascularization such as direct anastomosis (superficial temporal artery-to-middle cerebral artery), indirect anastomosis (encephaloduroarteriosynangiosis [EDAS] or encephaloduroarteriomyosynangiosis [EDAMS]), or combined bypass (direct and indirect simultaneously); 11) postoperative CT or MRI findings of cerebral infarction or hemorrhage; and 12) 6-month postoperative functional outcome using the mRS. For patients with newly diagnosed hyperlipidemia, medication was prescribed according to the "Guidelines for the management of dyslipidemia."12 Infarction or hemorrhage was diagnosed on initial CT or MRI only if the radiological finding developed acutely and was clinically symptomatic.

\section{Radiological Evaluation}

\section{Preoperative Evaluation}

Conventional CT, MRI, MR angiography, TFCA, and Diamox SPECT were performed in all patients preoperatively. Six independent, board-certified neuroradiologists reviewed radiological studies, including CT, MRI, and TFCA. Three independent, board-certified nuclear medicine radiologists reviewed SPECT. The Suzuki staging system is classified as stages I-VI according to the changes in moyamoya vessels observed on TFCA. ${ }^{2}$ We dichot- 
omized Suzuki stage into 2 groups: the early ICA stage group (Suzuki stages I-III) and the late ICA stage group (Suzuki stages IV-VI). Prior to the surgery, all patients underwent SPECT using 99m-technetium ethyl cysteinate dimer ( $99 \mathrm{~m} \mathrm{Tc}-\mathrm{ECD})$ to assess the basal cerebral blood flow and rCVR. Basal and acetazolamide (1 g, Diamox) stress

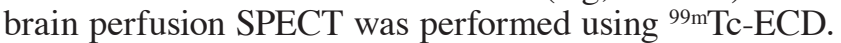
For a more detailed interpretation of SPECT, we modified a "10-bend" color scale..$^{13}$ In a 10-bend scale, normal cerebellar activity was set as the reference value, with values exceeding $90 \%$ perfusion shown in red. Local cerebral activity showing any gray matter below $60 \%$ of the reference value was determined as abnormal cerebral perfusion (shown in green, dark green, blue, and dark blue). If the color spectrum was diminished by at least one-third of the major vascular territories compared with the basal SPECT findings after administration of acetazolamide, the abnormal cerebral perfusion was defined as a reduced rCVR..$^{13}$ More specifically, a $10 \%$ decrease in rCVR after injecting acetazolamide, which was shown as reduction of one color spectrum from basal SPECT, was defined as mildly to moderately reduced. A greater than $20 \%$ reduction in rCVR that showed at least 2 color ranges decreased was moderately reduced. Moderately reduced with observation of steal phenomenon was defined as moderately to severely reduced. Symptomatic infarction or hemorrhage in a hemisphere was defined when localizing signs were shown on physical examination or there was a history of symptomatic transient ischemic attack corresponding to that affected hemisphere.

\section{Surgical Indications and Techniques}

Surgical revascularization was recommended when there was evidence of ischemic or hemorrhagic symptoms (transient ischemic attack, dysarthria, or hemiparesis) and hemodynamic compromise observed on SPECT. Surgery was performed in hemodynamically compromised patients exhibiting intracerebral hemorrhage or seizure. On the other hand, surgery was not recommended when there was evidence of recent infarct or hemorrhage (less than 6 weeks), a large cerebral infarction or encephalomalacia, or low Karnofsky Performance Scale score $(<60)$. In cases of surgery for the contralateral hemisphere, an interval of at least 6 weeks after the first surgery was recommended.

\section{Postoperative Management and Follow-Up}

Anemia and hypovolemia were strictly corrected in all patients. Systolic blood pressure was managed to be within normal range (100-140 mm Hg). Postoperative CT angiography was performed in all patients on the day of the operation. Nonenhanced CT was performed 1 day before discharge from the hospital and whenever infarct or hemorrhage was suspected. If the patient developed new neurological deficits but a negative finding was observed on CT, immediate diffusion-weighted imaging was recommended to assess the acute cerebral infarction. The evidence of infarction or hemorrhage within 2 weeks of operation was defined as postoperative infarction or hemorrhage. The clinical outcomes were analyzed using the mRS score 6 months after surgical revascularization. The outcome was dichotomized into favorable $(\mathrm{mRS}$ score $<3$ ) and unfavorable (mRS score $\geq 3$ ).

\section{Statistical Analysis}

Qualitative factors were analyzed using a standard chisquare test or Fisher's exact test, and quantitative factors were analyzed using an unpaired Student t-test or MannWhitney U-test. Univariate and multivariate logistic regressions adopting a backward elimination technique were conducted to evaluate the potential risk factors associated with a postoperative unfavorable result.

\section{Optimization of Scoring System}

The scoring system model development was achieved exclusively from the development cohort. Univariate and multivariable logistic regressions with generalized estimating equations (GEEs) that clarified the clustering from the same subject were performed to evaluate the risk variables of unfavorable outcome after surgery in the development cohort. The prediction model was developed based on a multivariable logistic regression analysis using backward elimination on 1000 bootstrap samples. Variables involved in the logistic regression model were selected as predictors when included at least $50 \%$ of the time. Factors showing a lower than $50 \%$ relative selection frequency were eliminated from the final model. The tolerance and variance inflation factor of each covariate were evaluated to examine the collinearity between the covariates of the final multivariable model. A point scoring system was developed to predict functional outcomes based on the $\beta$-coefficients from the final model.

\section{Grading With the Scoring System}

The proportional weight for each variable in the scoring system was evaluated adopting the variable's $\beta$-coefficients from multivariate logistic regression analysis with GEE. The minimum $\beta$ estimate was used as the reference value by which all other variables were calculated. The raw score was developed with a summed weighted score of each component. The scoring system was stratified into 3 groups: low, intermediate, and high risk. The analysis of the risk group stratification was achieved via optimization adopting receiver operating characteristic (ROC) curve analysis and the Hosmer-Lemeshow goodness-of-fit test. ${ }^{14}$

\section{Validation of the Scoring System}

The scoring system was validated in development, internal, temporal, and external validation cohorts by assessing model discrimination and calibration. The Cstatistic was adopted for the quantification of discrimination, which is equal to the area under the ROC. Calibration was determined by the Hosmer-Lemeshow goodnessof-fit test. The data set from the development cohort was obtained for the evaluation of the internal validity in the scoring system. The bootstrap design with 1000 bootstrap resamples was adopted for the adjustment of the optimism, which was obtained from the same data set used to develop the prediction model. The optimism-corrected C-statistics were determined by estimating the amount of deduction in optimism (the Harrell validate function was 
used for the evaluation) from the apparent C-statistic in the development cohort.

Statistical analysis was performed using IBM SPSS (version 21, IBM Corp.), SAS (version 9.4, SAS Institute), and R software (version 3.6.0; www.r-project.org) in association with packages rms and $\mathrm{pROC} ; \mathrm{p}<0.05$ indicated statistical significance. The precision of the estimates was assessed with $95 \%$ confidence intervals.

\section{Data Availability}

Data from the current study, including data supporting the findings of our study, are accessible from the corresponding author on request.

\section{Results}

\section{Characteristics of the Development and Validation Cohorts}

We retrospectively identified 302 MMD patients who underwent revascularization surgery between January 1998 and December 2016. Patients with moyamoya syndrome $(\mathrm{n}=18)$ were excluded from our study (Table 1$)$. Seven patients were lost to follow-up prior to 6 months postoperatively. An additional 17 patients were excluded, as they had insufficient radiological data and underlying causes such as trauma, cancer, or pneumonia that may have affected the functional outcome. Finally, 260 MMD patients were included in our study, accounting for 321 hemispheres (Fig. 1). The mean patient age was $37.2 \pm 12$ years (range 18-68 years) with a female predominance (213 hemispheres, 66.4\%). The most common comorbidities were hypertension (29.9\%) and hyperlipidemia $(10.6 \%)$. Cigarette smoking was identified in 83 cases (25.9\%), and there were 29 cases (9\%) of first-degree relatives diagnosed with MMD. The same inclusion and exclusion criteria were applied for the temporal and external validation cohorts from multicenter medical institutions between January 2017 and October 2018, for a total of 69 patients with 78 hemispheres (Fig. 2).

\section{Development Cohort}

\section{Preoperative Radiological Evaluation and Clinical Symptoms}

The preoperative $\mathrm{mRS}$ score was $<3$ in 247 cases (76.9\%). Extremity weakness or paresthesia was the most common initial presentation (39.6\%), followed by headache $(21.8 \%)$ and dysarthria (20.6\%). Symptomatic cerebral infarction was observed in 177 cases (55.1\%) on CT or MRI at admission, whereas 37 cases $(11.5 \%)$ had evidence of intracranial hemorrhage. The remaining cases (33.3\%) had no evidence of infarction or hemorrhage. There were more cases of Suzuki stage $\geq$ IV (62.3\%) and PCA involvement $(62.9 \%)$ as noted on TFCA. More than half of the cases had mildly to moderately reduced rCVR (54.2\%) on Diamox SPECT.

\section{Surgery and Functional Outcome}

Combined bypass was most commonly performed (44.2\%), followed by direct (29\%) and indirect (26.8\%) bypasses. Postoperative cerebral infarction was diagnosed in 36 cases, and hemorrhage was diagnosed in 51 cases.
TABLE 1. Characteristics of the development cohort

\begin{tabular}{|c|c|c|}
\hline Characteristic & $\begin{array}{l}\text { Patients } \\
(n=260)\end{array}$ & $\begin{array}{c}\text { Hemispheres } \\
\quad(n=321)\end{array}$ \\
\hline Mean age, yrs & $38 \pm 12.1$ & $37.2 \pm 12$ \\
\hline Female sex & $172(66.2 \%)$ & $213(66.4 \%)$ \\
\hline \multicolumn{3}{|l|}{ Comorbidity } \\
\hline Hypertension & $76(29.2 \%)$ & $96(29.9 \%)$ \\
\hline Diabetes & $21(8.1 \%)$ & $23(7.2 \%)$ \\
\hline Hyperlipidemia & $28(10.8 \%)$ & $34(10.6 \%)$ \\
\hline $\begin{array}{l}\text { Ischemic heart disease (angina } \\
\text { or MI) }\end{array}$ & $3(1.2 \%)$ & $4(1.2 \%)$ \\
\hline Smoking & $72(27.7 \%)$ & $83(25.9 \%)$ \\
\hline MMD family history & $24(9.2 \%)$ & $29(9 \%)$ \\
\hline \multicolumn{3}{|l|}{ No. of surgical revascularizations } \\
\hline 1 & $202(77.7 \%)$ & \\
\hline 2 & $55(21.2 \%)$ & \\
\hline 3 & $3(1.2 \%)$ & \\
\hline \multicolumn{3}{|l|}{ Preop functional status } \\
\hline $\mathrm{mRS}<3$ & & $247(76.9 \%)$ \\
\hline$m R S \geq 3$ & & $74(23.1 \%)$ \\
\hline \multicolumn{3}{|l|}{ Initial presentation } \\
\hline Headache & & $70(21.8 \%)$ \\
\hline Dizziness & & $20(6.2 \%)$ \\
\hline Dysarthria & & $66(20.6 \%)$ \\
\hline Extremity weakness or paresthesia & & $127(39.6 \%)$ \\
\hline Seizure/LOC & & $17(5.3 \%)$ \\
\hline Visual deficit & & $21(6.5 \%)$ \\
\hline \multicolumn{3}{|l|}{ Initial CT or MRI finding* } \\
\hline Infarction & & $177(55.1 \%)$ \\
\hline Hemorrhage & & $37(11.5 \%)$ \\
\hline \multicolumn{3}{|l|}{ Suzuki stage } \\
\hline$<I V$ & & $121(37.7 \%)$ \\
\hline$\geq \mathrm{IV}$ & & $200(62.3 \%)$ \\
\hline \multicolumn{3}{|l|}{ PCA involvement } \\
\hline No & & $119(37.1 \%)$ \\
\hline Yes & & $202(62.9 \%)$ \\
\hline \multicolumn{3}{|l|}{ rCVR findings on Diamox SPECT } \\
\hline Mildly to moderately reduced & & $174(54.2 \%)$ \\
\hline Moderately reduced & & $116(36.1 \%)$ \\
\hline Moderately to severely reduced & & $31(9.7 \%)$ \\
\hline \multicolumn{3}{|l|}{ Type of surgical revascularization } \\
\hline Indirect bypass (EDAMS or EDAS) & & $86(26.8 \%)$ \\
\hline Direct bypass & & $93(29 \%)$ \\
\hline Combined bypass & & $142(44.2 \%)$ \\
\hline \multicolumn{3}{|l|}{ Postop CT or MRI } \\
\hline Infarction & & $36(11.2 \%)$ \\
\hline Hemorrhage & & $51(15.9 \%)$ \\
\hline \multicolumn{3}{|l|}{ Postop 6-mo functional outcome } \\
\hline $\mathrm{mRS}<3$ & & $290(90.3 \%)$ \\
\hline$m R S \geq 3$ & & $31(9.7 \%)$ \\
\hline
\end{tabular}

LOC = loss of consciousness; $\mathrm{MI}=$ myocardial infarction.

* Includes symptomatic cerebral infarction or hemorrhage.

Among these cases, 6 patients showed both postoperative cerebral infarction and hemorrhage and they were assigned to a more clinically correlated group. Among 260 


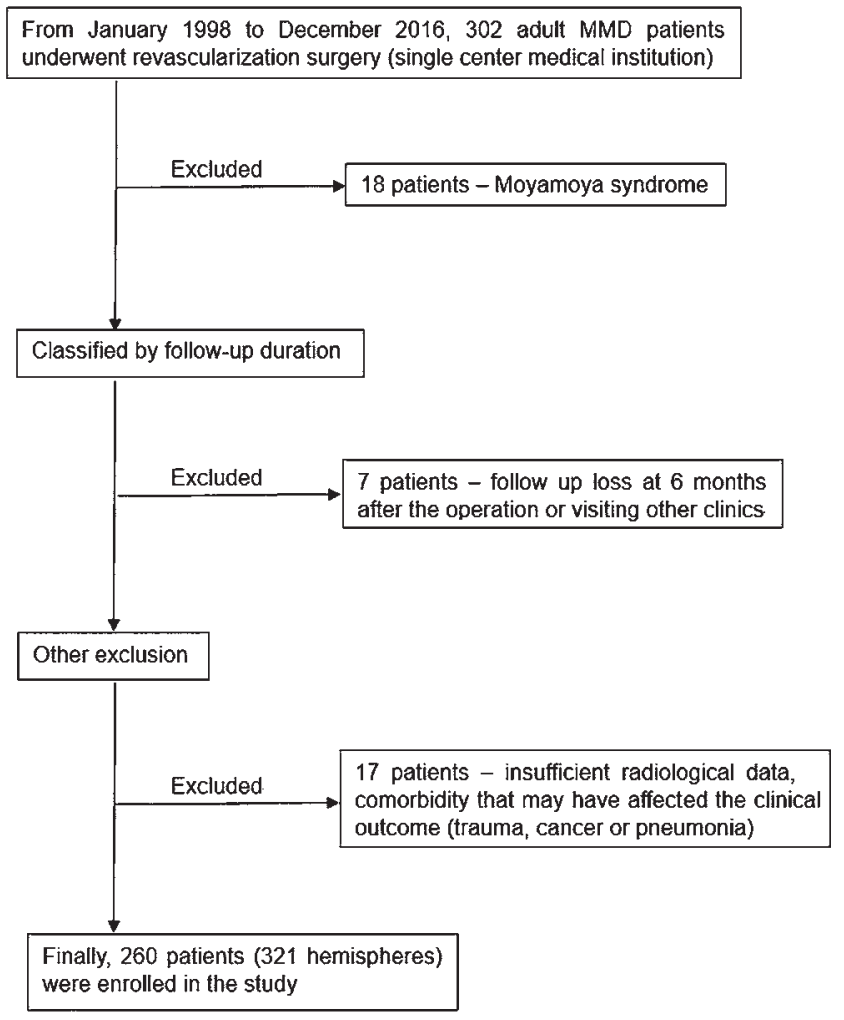

FIG. 1. Flowchart showing patient inclusion and exclusion for development cohort.

patients, $202(77.7 \%)$ and $55(21.2 \%)$ underwent surgical revascularization on one and both hemispheres, respectively. The remaining 3 patients $(1.2 \%)$ underwent repeat vascularization surgery on the same hemisphere due to newly developed symptoms and hemodynamic compromise despite previous bypass surgery. In these patients, the surgical methods used during repeat revascularization were different from those performed during the previous surgery.

All patients underwent assessment of functional outcome 6 months postoperatively using the mRS. Most cases $(290,90.3 \%)$ showed favorable outcome, whereas unfavorable outcome was identified in 31 cases $(9.7 \%)$.

\section{Statistical Analysis of Risk Factors}

For the univariate analysis, hyperlipidemia $(\mathrm{p}<0.001)$, smoking $(\mathrm{p}=0.001)$, preoperative infarction on CT or MRI $(\mathrm{p}<0.001)$, and Diamox SPECT (moderately reduced, $\mathrm{p}=$ 0.004 and moderately to severely reduced, $\mathrm{p}<0.001$ ) were determined to be the factors associated with unfavorable outcome. Univariate analysis showing variables with $\mathrm{p}<$ 0.05 and those considered to be clinically relevant were included in the multivariate analysis. Among these variables, hyperlipidemia (OR 5.78, 95\% CI 2.40-13.93; p < 0.001 ), smoking (OR 2.41, 95\% CI 1.11-5.25; $\mathrm{p}=0.026$ ), cerebral infarction on preoperative CT or MRI (OR 6.75, 95\% CI 2.42-18.86; p < 0.001), and Diamox SPECT results of mildly to moderately reduced as reference value, moderately reduced $(\mathrm{OR} 1.93,95 \%$ CI $0.76-4.90 ; \mathrm{p}=$

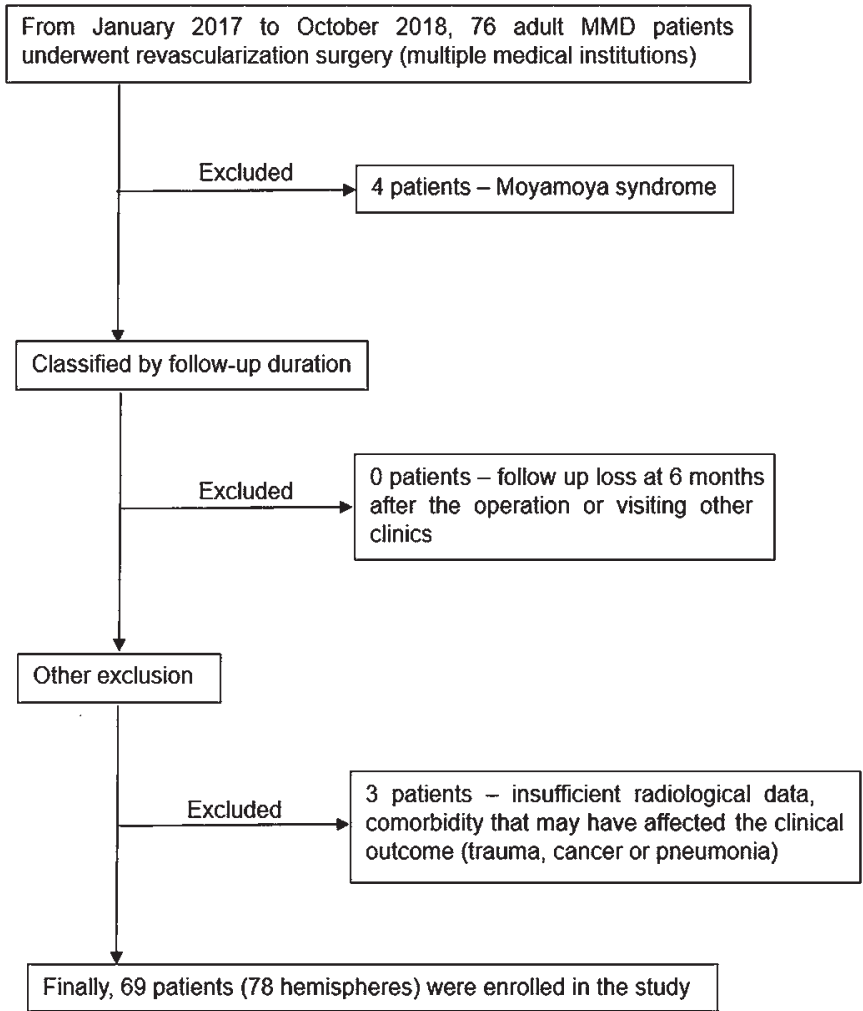

FIG. 2. Flowchart showing patient inclusion and exclusion for temporal and external validation cohorts.

0.168 ), and moderately to severely reduced (OR 4.26, 95\% CI $1.46-12.39 ; \mathrm{p}=0.008$ ) were analyzed to be independent risk factors for unfavorable outcome postoperatively (Table 2).

\section{Development of a Scoring System}

The independent risk factors from the univariate and multivariate analyses were scaled for the odds ratio using the relative weighting method to evaluate the unfavorable outcome. These 4 variables were then incorporated into a clinical prediction tool with associated point values, as the estimate value for moderately reduced rCVR on Diamox SPECT was converted to 1 as reference value, and other values were converted accordingly. Evidence of hyperlipidemia was assigned 3 points, and the score was 0 if absent. One point was assigned for smokers and 0 points for nonsmokers. Three points were given for evidence of cerebral infarction on preoperative CT or MRI and 0 points if absent. Preoperative Diamox SPECT results with mildly to moderately reduced, moderately reduced, and moderately to severely reduced rCVR were assigned 0,1 , and 2 points, respectively. These scores were summed to determine the hemisphere's prediction score of outcome, and thus the scoring system ranged from 0 to 9 (Table 3).

\section{Risk Group Stratification and Estimates for Unfavorable Outcome}

After development of the scoring system, we strati- 
TABLE 2. Univariate and multivariate analyses of clinical factors for unfavorable outcome from the development cohort

\begin{tabular}{|c|c|c|c|c|c|}
\hline \multirow[b]{2}{*}{ Parameter } & \multirow{2}{*}{$\begin{array}{c}\text { No. of } \\
\text { Hemispheres }\end{array}$} & \multicolumn{2}{|c|}{ Univariate Analysis } & \multicolumn{2}{|c|}{ Multivariate Analysis } \\
\hline & & $\mathrm{OR}(95 \% \mathrm{Cl})$ & $p$ Value & $\mathrm{OR}(95 \% \mathrm{Cl})$ & $\mathrm{p}$ Value \\
\hline Female sex & 213 & $0.51(0.26-1.01)$ & 0.054 & & \\
\hline \multicolumn{6}{|l|}{ Comorbidity } \\
\hline Hypertension & 96 & $1.78(0.87-3.62)$ & 0.113 & & \\
\hline Diabetes & 23 & $2.67(0.93-7.66)$ & 0.068 & & \\
\hline Hyperlipidemia & 34 & $7.97(3.34-19.04)$ & $<0.001$ & $5.78(2.40-13.93)$ & $<0.001$ \\
\hline Ischemic heart disease (angina or MI) & 4 & $1.87(0.19-22.07)$ & 0.620 & & \\
\hline Smoking & 83 & $3.47(1.71-7.02)$ & 0.001 & $2.42(1.11-5.25)$ & 0.026 \\
\hline MMD family history & 29 & $0.18(0.02-1.40)$ & & & \\
\hline \multicolumn{6}{|l|}{ Initial presentation } \\
\hline Headache & 70 & $0.57(0.26-1.25)$ & 0.157 & & \\
\hline Dizziness & 20 & $1.06(0.43-2.63)$ & 0.902 & & \\
\hline Dysarthria & 66 & $0.93(0.43-1.99)$ & 0.845 & & \\
\hline Extremity weakness or paresthesia & 127 & $0.85(0.41-1.76)$ & 0.665 & & \\
\hline Seizure/LOC & 17 & $0.98(0.20-4.85)$ & 0.979 & & \\
\hline Visual deficit & 21 & $1.84(0.77-4.38)$ & 0.169 & & \\
\hline \multicolumn{6}{|l|}{ Initial CT or MRI findings } \\
\hline Infarction & 177 & $7.38(2.97-18.36)$ & $<0.001$ & $6.75(2.42-18.86)$ & $<0.001$ \\
\hline Hemorrhage & 37 & $1.09(0.41-2.84)$ & 0.868 & & \\
\hline \multicolumn{6}{|l|}{ Suzuki stage } \\
\hline$<\mathrm{IV}$ & 121 & 1 & & & \\
\hline$\geq \mathrm{IV}$ & 200 & $1.62(0.77-3.43)$ & 0.207 & & \\
\hline \multicolumn{6}{|l|}{ PCA involvement } \\
\hline No & 119 & 1 & & & \\
\hline Yes & 202 & $1.10(0.66-2.97)$ & 0.376 & & \\
\hline \multicolumn{6}{|l|}{ rCVR findings on Diamox SPECT } \\
\hline Mildly to moderately reduced & 174 & 1 & 0.001 & 1 & 0.061 \\
\hline Moderately reduced & 116 & $3.40(1.48-7.84)$ & 0.004 & $1.93(0.76-4.90)$ & 0.168 \\
\hline Moderately to severely reduced & 31 & $6.81(2.49-18.67)$ & $<0.001$ & $4.26(1.46-12.39)$ & 0.008 \\
\hline \multicolumn{6}{|l|}{ Type of surgical revascularization } \\
\hline Indirect bypass (EDAMS or EDAS) & 86 & 1 & 0.566 & & \\
\hline Direct bypass & 93 & $1.48(0.61-3.60)$ & 0.388 & & \\
\hline Combined bypass & 142 & $0.95(0.43-2.13)$ & 0.906 & & \\
\hline
\end{tabular}

fied each evaluated hemisphere into 3 risk group grades based on the total scoring system: low risk, 0-3 points; intermediate risk, 4-6 points; and high risk, 7-9 points (Table 4). Considering that our scoring system is based on calculation of one hemisphere only, two different scores and grades may be possible for patients with bilateral surgery. According to our scoring system, estimates of mRS scores greater than 3 increase as the total score increases; when the point was scored 0,1 , and 2 , the estimates of mRS score greater than 3 were $1.51 \%, 2.87 \%$, and $5.38 \%$, respectively. When the score using our system was 9 , the estimate of an unfavorable outcome was $84.9 \%$. According to this risk group stratification, the development cohort of 321 cases was categorized as follows: 197 (61.37\%) as low risk, $103(32.09 \%)$ as intermediate risk, and 21 (6.54\%) as high risk. Generally, when the score was lower, the estimate of the mRS score greater than 3 was low. Conversely, the estimates of unfavorable outcome were much higher when the score was high. Similar findings were observed in the validation cohort. According to our risk group stratification, 63 (80.77\%), 11 (14.1\%), and 4 (5.13\%) cases were assigned to low-, intermediate-, and high-risk groups, respectively. Among these cases, 7 cases $(8.97 \%)$ had unfavorable outcomes.

\section{Assessment of the Scoring System Model}

To assess the performance of our prediction model, we adopted C-statistics to evaluate the model's discrimination and Hosmer-Lemeshow goodness-of-fit test for the calibration of the model. Our model showed good discrimination and calibration among development $(81.2 \%, 95 \% \mathrm{CI}$ $0.743-0.881 ; \mathrm{p}=0.568)$, internal validation $(79.9 \%, 95 \%$ CI $0.730-0.868)$, and external validation $(95.4 \%, 95 \%$ CI $0.896-1 ; p=0.097$ ) cohorts (Table 5). The ROC curve of prediction for the scoring system in the development and temporal and external validation cohorts is also illustrated (Fig. 3). 
TABLE 3. Development of scoring system for prediction of unfavorable outcome

\begin{tabular}{lcc}
\hline \multicolumn{1}{c}{ Parameter } & Estimate & Points \\
\hline Intercept & -4.718 & \\
\hline Comorbidity: hyperlipidemia & & \\
\hline No & & 0 \\
\hline Yes & 1.754 & 3 \\
\hline Smoking & & \\
\hline No & 0.882 & 1 \\
\hline Yes & & \\
\hline Initial CT or MRI: infarction & & 0 \\
\hline No & 1.909 & 3 \\
\hline Yes & & \\
\hline rCVR findings on Diamox SPECT & & 0 \\
\hline Mildly to moderately reduced & 0.656 & 1 \\
\hline Moderately reduced & 1.448 & 2 \\
\hline Moderately to severely reduced & & 9 \\
\hline Total &
\end{tabular}

\section{Discussion}

MMD is heterogeneous in clinical presentation, progression, and response to treatment. Hence, we believe that TFCA studies alone may not provide sufficient information to comprehend the MMD pathophysiology. According to Suzuki and Takaku, ${ }^{2}$ the appearance of moyamoya vessels is not observed on angiograms of the most severe MMD, implicating that moyamoya vessels disappear with the progression of disease severity. In addition, the Suzuki stage does not take into account clinical severity or risk factors of MMD., ${ }^{2,10}$ In the early stage of MMD, proximal ICA occlusion may be compensated for by robust external carotid artery, pial, and leptomeningeal collaterals; hence, the patient may have only mild symptoms. Uncompensated mild ICA stenosis may result in severe clinical symptoms in the later stage of MMD. ${ }^{15}$ Therefore, the Suzuki stage has limited value in terms of clinical correlation. We believe that the treatment decision of MMD is frequently based on an individual surgeon's training and expertise, partly because of a lack of reliable data on which to base decisions and MMD's vague prognosis. In this context, individualized treatment decisions are limited. Hence, a prediction tool to estimate the outcome of MMD in combination with multimodality assessment is necessary to achieve the best outcome.

The pathogenesis of MMD is unclear, and evaluation of comorbidities may be helpful in determining its clinical prognosis. According to a study from the Mayo Clinic in Minnesota between 1979 and 2011, the incidence of hyperlipidemia in MMD was significantly increased in comparison with the general population $(27.7 \%$ vs $16.3 \%) .{ }^{16}$ In this study, hyperlipidemia was the most prevalent comorbidity, overtaking hypertension, smoking, and type 2 diabetes. Another large study of patients with MMD who were treated between 1979 and 2011 also reported a high incidence of hyperlipidemia (37.3\%). ${ }^{17}$ The reason for this significantly higher rate of hyperlipidemia is not clear, although it is a known association with other occlusive vascular pathology and untreated hypothyroidism. ${ }^{18}$ Interestingly, despite its high prevalence, many studies focusing on the prognosis of MMD did not include cholesterol values. ${ }^{69}$ Some studies included lipid profiles, but the analysis showed less significant results. ${ }^{5,8}$ These conflicting results may be due to different lipid parameters and the levels of cholesterol involved in various studies. The association between dyslipidemia and stroke risk is intricate and appears to be different, mainly due to the stroke subtype, lipid parameters, and cholesterol level. ${ }^{19}$ Accordingly, various lipid parameters and cholesterol levels adopted in different studies resulted in conflicting results for interpretation of MMD outcome.

Smoking is a well-known independent risk factor for incident and recurrent stroke..$^{20}$ One study urged that smoking is an important risk factor for rebleeding in MMD

TABLE 4. Risk group stratification and estimates of unfavorable outcome

\begin{tabular}{|c|c|c|c|c|c|c|}
\hline \multirow[b]{2}{*}{ Risk Group } & \multirow[b]{2}{*}{ Points } & \multirow{2}{*}{$\begin{array}{c}\text { Estimate of mRS } \\
\quad \geq 3 \text { Risk }\end{array}$} & \multicolumn{2}{|c|}{ Development Cohort $(n=321)$} & \multicolumn{2}{|c|}{ Validation Cohort $(n=78)^{*}$} \\
\hline & & & No. of Hemispheres (\%) & No. $w / m R S \geq 3(\%)$ & No. of Hemispheres (\%) & No. $w / m R S \geq 3(\%)$ \\
\hline \multirow{4}{*}{ Low } & 0 & 0.0151 & $75(23.4)$ & $1(0.013)$ & $30(38.5)$ & $0(0)$ \\
\hline & 1 & 0.0287 & $40(12.5)$ & $2(0.05)$ & $21(26.9)$ & $0(0)$ \\
\hline & 2 & 0.0538 & $16(5)$ & $0(0)$ & $3(3.8)$ & $1(0.33)$ \\
\hline & 3 & 0.0989 & $66(20.6)$ & $3(0.045)$ & $9(11.5)$ & $0(0)$ \\
\hline \multirow{3}{*}{ Intermediate } & 4 & 0.1745 & $61(19)$ & $6(0.098)$ & $7(9)$ & $1(0.143)$ \\
\hline & 5 & 0.2895 & $33(10.3)$ & $4(0.121)$ & $2(2.6)$ & $1(0.5)$ \\
\hline & 6 & 0.4399 & $9(2.8)$ & $4(0.444)$ & $2(2.6)$ & $1(0.5)$ \\
\hline \multirow{3}{*}{ High } & 7 & 0.6022 & $9(2.8)$ & $4(0.444)$ & $1(1.3)$ & $1(100)$ \\
\hline & 8 & 0.747 & $10(3.1)$ & $6(0.6)$ & $3(3.8)$ & $2(0.667)$ \\
\hline & 9 & 0.8490 & $2(0.6)$ & $1(0.5)$ & 0 & $0(0)$ \\
\hline Total & & & $321(100)$ & $31(9.7)$ & $78(100)$ & $7(8.97)$ \\
\hline
\end{tabular}

*Validation cohort = temporal validation cohort + external validation cohort. 
TABLE 5. Assessment of scoring system

\begin{tabular}{lccc}
\hline & Development Cohort $(\mathrm{n}=321)$ & Internal Validation Cohort $(\mathrm{n}=321)$ & Temporal \& External Validation Cohort $(\mathrm{n}=78)$ \\
\hline C-statistic $(95 \% \mathrm{Cl})$ & $0.812(0.743-0.881)$ & $0.799(0.730-0.868)$ & $0.954(0.896-1)$ \\
\hline $\mathrm{HL}$ goodness-of-fit test & & & 6.313 \\
\hline$\chi^{2}$ & 2.938 & 3 & 0.097 \\
\hline Degrees of freedom & 4 & & \\
\hline p value & 0.568 & & \\
\hline
\end{tabular}

$\mathrm{HL}=$ Hosmer-Lemeshow.

and thereby contributed poor outcome. ${ }^{21}$ Another study showed that when MMD patients stopped or reduced smoking, stabilization of symptoms resulted, whereas patients who continued to smoke had progressive symptoms for 10 years. ${ }^{22}$ As shown in our study, smoking and hyperlipidemia are the only modifiable factors that may have significant clinical impact on outcome. More importantly, smoking may raise the risk of ischemic stroke, which is increased by hyperlipidemia. ${ }^{23}$ Accordingly, we believe that the fundamental strategies for MMD should be focused not only on medical or surgical treatment but also on education of a lifestyle modification, such as adequate diet, increase in physical activity, smoking cessation, and weight loss in obese patients, which should be recommended to MMD patients as soon as possible.

Various risk factors are known to have an impact on postoperative outcome in MMD patients, including female sex, ${ }^{24}$ perioperative hypotension, ${ }^{25}$ diabetes, ${ }^{8}$ preoperative infarction, ${ }^{7,9}$ and PCA involvement., ${ }^{7,25}$ The controversy over which factors contribute as risk factors remains

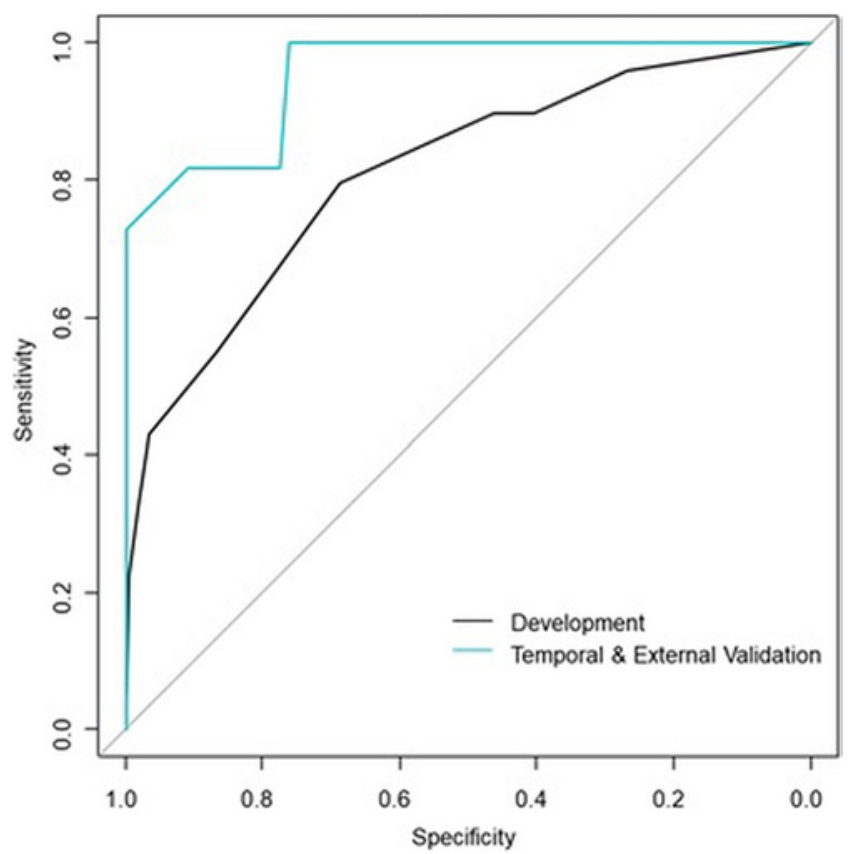

FIG. 3. ROC curve of prediction for the scoring system in the development and temporal and external validation cohorts (C-statistics $=0.812$ vs 0.954$)$. Figure is available in color online only. unclear in the literature. Park et al. showed a significant difference in postoperative infarction rates between preoperative infarction and noninfarction groups and hence concluded that preoperative cerebral infarction history may result in a more unfavorable outcome in MMD? ${ }^{7}$ A similar finding was observed by Funaki et al. that cerebral infarction as a presenting symptom in MMD was associated with much higher rates of perioperative ischemic complications compared with patients with stable MMD. ${ }^{26}$ Patients with a preoperative infarction seem to have a lack of collaterals to compensate for hemodynamic deterioration. ${ }^{9}$ One possible cause of this phenomenon is that these patients are likely to have very weak and fragile vessels and hence intraoperative procedures may have caused stress on hemodynamic regulation, thereby resulting in unfavorable outcome.

Previous studies focusing on a prediction model for MMD were largely dependent on angiographic studies. ${ }^{5-9}$ However, as previously mentioned, there is often a discrepancy between clinical severity and angiographic results, which may be due to the complex interaction among various patient factors, including patient history, comorbidities, cerebrovascular flow and reserve, and collateral network. Accordingly, we exclusively developed a scoring system to overcome these issues. Laboratory blood samples, CT scans, and MR images can be easily obtained from medical centers where MMD is diagnosed and treated. However, different institutions used different modalities to evaluate cerebral hemodynamics. The most common modalities used to assess brain hemodynamics are Xe-enhanced CT, PET, MRI with dynamic contrast, and SPECT. ${ }^{27}$ The advantage of Xe-enhanced CT and PET is the quantified evaluation of hemodynamic compromise, consequently avoiding the obstacles of inconclusive measurement results if both hemispheres show hemodynamic impairment. However, Xe-CT also has some limitations such as motion artifacts and patient discomfort during the inhalation of xenon. ${ }^{27}$ PET was not included in our study, as it is much less available and relatively more expensive than other modalities. ${ }^{27}$ SPECT has some limitations, such that it has less optimal spatial resolution, but it has been commonly used in MMD patients in conjunction with acetazolamide challenge to precisely assess cerebrovascular reserve. ${ }^{27}$ Also, serial SPECT studies are excellent in predicting symptomatic hyperperfusion and avoiding permanent neurological sequelae postoperatively. ${ }^{28}$

The key objective of our study was the development of an MMD scoring system for prediction of postoperative outcome. This scoring system is not solely based on 
angiographic studies but also includes the patient's social history and comorbidities and multimodal imaging tools with external validation to show its generalizability and reliability. According to our proposed scoring system, low-, intermediate-, and high-risk grades may be distinguished preoperatively with an increasing risk of unfavorable outcome postoperatively. Although a few studies have evaluated risk factors of MMD or propose a scoring system, these systems were mainly focused on radiological results. ${ }^{5-9,29,30}$ Moreover, the efficiency of these scoring systems was not externally validated. To the best of our knowledge, the current study is the first to address the scoring system of MMD preoperatively, using various parameters to predict postoperative outcome in relatively large internal and external cohorts and validate its clinical value.

Referral bias cannot be excluded, as all participating centers in our study were large university-affiliated tertiary care institutions. Consequently, our scoring system may not represent the general population. A lack of information on pre- and postoperative medical management is another limitation. Different institutions may have different protocols for blood pressure and intracranial pressure management, which may play a significant role. Some patients underwent a single-hemisphere operation, and the revascularization surgery may have improved collateral flow on the operated hemisphere and hence the untreated contralateral hemisphere may have had a clinical impact on the stroke risk. ${ }^{31}$ Regardless of these limitations, our study included a comparatively large sample size to develop a scoring system that was validated both internally and externally in a prospective nature to show its generalizability and efficiency, which has never been described in a study. Another strength is that even though all cases underwent revascularization surgery in the present study, the parameters related to the scoring system can be evaluated preoperatively. Hence, the application of our presented scoring system in anticipating clinical outcome does not solely rely on the surgical factors and thus can also be recommended to MMD patients who are not candidates for the surgery.

\section{Conclusions}

Our data revealed the importance of cigarette smoking and hyperlipidemia that may have a greater impact on clinical outcome than we expected. These parameters are the only modifiable factors, and hence lifestyle modification with education should be recommended to MMD patients as early as possible. It is true that a simple scoring system as proposed in our study cannot precisely evaluate all variable factors of a disease as intricate as MMD. However, in combination, our results offer novel insights into the composition of moyamoya population and may help guide physicians in developing a treatment plan and predict the prognosis.

\section{Acknowledgments}

This work was supported by the National Research Foundation of Korea (NRF) grant funded by the Korea government (MSIT) (NRF-NRF-2017R1D1A1B04035927) and 2018-779, 2019IL0818, from the Asan Institute for Life Sciences, Asan Medical Center, Seoul, Korea.

\section{References}

1. Scott RM, Smith ER. Moyamoya disease and moyamoya syndrome. N Engl J Med. 2009;360(12):1226-1237.

2. Suzuki J, Takaku A. Cerebrovascular "moyamoya" disease. Disease showing abnormal net-like vessels in base of brain. Arch Neurol. 1969;20(3):288-299.

3. Czabanka M, Peña-Tapia P, Schubert GA, et al. Proposal for a new grading of moyamoya disease in adult patients. Cerebrovasc Dis. 2011;32(1):41-50.

4. Kim DS, Huh PW, Kim HS, et al. Surgical treatment of moyamoya disease in adults: combined direct and indirect vs. indirect bypass surgery. Neurol Med Chir (Tokyo). 2012;52(5):333-338.

5. Ge P, Zhang Q, Ye X, et al. Clinical features, surgical treatment, and long-term outcome in elderly patients with moyamoya disease. World Neurosurg. 2017;100:459-466.

6. Kim T, Oh CW, Kwon OK, et al. Stroke prevention by direct revascularization for patients with adult-onset moyamoya disease presenting with ischemia.J Neurosurg. 2016;124(6):1788-1793.

7. Park W, Ahn JS, Lee HS, et al. Risk factors for newly developed cerebral infarction after surgical revascularization for adults with moyamoya disease. World Neurosurg. 2016:92:65-73.

8. Zhao M, Deng X, Gao F, et al. Ischemic stroke in young adults with moyamoya disease: prognostic factors for stroke recurrence and functional outcome after revascularization. World Neurosurg. 2017;103:161-167.

9. Zhao M, Deng X, Zhang D, et al. Risk factors for and outcomes of postoperative complications in adult patients with moyamoya disease. J Neurosurg. 2019;130(2):531-542.

10. Fukui M. Guidelines for the diagnosis and treatment of spontaneous occlusion of the circle of Willis ('moyamoya' disease). Research Committee on Spontaneous Occlusion of the Circle of Willis (Moyamoya Disease) of the Ministry of Health and Welfare, Japan. Clin Neurol Neurosurg. 1997;99(suppl 2):S238-S240.

11. Hashimoto N, Miyamoto S, Nagata I, et al. Guidelines for diagnosis and treatment of moyamoya disease (spontaneous occlusion of the circle of Willis). Neurol Med Chir (Tokyo). 2012;52(5):245-266.

12. Rhee EJ, Kim HC, Kim JH, et al. 2018 Guidelines for the management of dyslipidemia. Korean J Intern Med. 2019;34(4):723-771.

13. Kim JS, Moon DH, Kim GE, et al. Acetazolamide stress brain-perfusion SPECT predicts the need for carotid shunting during carotid endarterectomy. J Nucl Med. 2000;41(11):1836-1841.

14. Hosmer DW, Lemeshow S. Goodness of fit tests for the multiple logistic regression model. Commun Stat Theory Methods. 1980;9(10):1043-1069.

15. Strother MK, Anderson MD, Singer RJ, et al. Cerebrovascular collaterals correlate with disease severity in adult North American patients with moyamoya disease. AJNR Am J Neuroradiol. 2014;35(7):1318-1324.

16. Bower RS, Mallory GW, Nwojo M, et al. Moyamoya disease in a primarily white, midwestern US population: increased prevalence of autoimmune disease. Stroke. 2013;44(7):1997-1999.

17. Wei YC, Liu CH, Chang TY, et al. Coexisting diseases of moyamoya vasculopathy. J Stroke Cerebrovasc Dis. 2014;23(6):1344-1350.

18. Squizzato A, Gerdes VE, Brandjes DP, et al. Thyroid diseases and cerebrovascular disease. Stroke. 2005;36(10):2302-2310.

19. Pol T, Held C, Westerbergh J, et al. Dyslipidemia and risk of cardiovascular events in patients with atrial fibrillation 
treated with oral anticoagulation therapy: insights from the ARISTOTLE (Apixaban for Reduction in Stroke and Other Thromboembolic Events in Atrial Fibrillation) trial. J Am Heart Assoc. 2018;7(3):e007444.

20. Shinton R, Beevers G. Meta-analysis of relation between cigarette smoking and stroke. BMJ. 1989;298(6676):789-794.

21. Kang S, Liu X, Zhang D, et al. Natural course of moyamoya disease in patients with prior hemorrhagic stroke. Stroke. 2019;50(5):1060-1066.

22. Levine SR, Fagan SC, Pessin MS, et al. Accelerated intracranial occlusive disease, oral contraceptives, and cigarette use. Neurology. 1991;41(12):1893-1901.

23. Hata J, Doi Y, Ninomiya T, et al. Combined effects of smoking and hypercholesterolemia on the risk of stroke and coronary heart disease in Japanese: the Hisayama study. Cerebrovasc Dis. 2011;31(5):477-484

24. Khan N, Achrol AS, Guzman R, et al. Sex differences in clinical presentation and treatment outcomes in moyamoya disease. Neurosurgery. 2012;71(3):587-593.

25. Jung YJ, Ahn JS, Kwon DH, Kwun BD. Ischemic complications occurring in the contralateral hemisphere after surgical treatment of adults with moyamoya disease. J Korean Neurosurg Soc. 2011;50(6):492-496.

26. Funaki T, Takahashi JC, Takagi Y, et al. Unstable moyamoya disease: clinical features and impact on perioperative ischemic complications. J Neurosurg. 2015;122(2):400-407.

27. Lee M, Zaharchuk G, Guzman R, et al. Quantitative hemodynamic studies in moyamoya disease: a review. Neurosurg Focus. 2009;26(4):E5.

28. Uchino H, Kuroda S, Hirata K, et al. Predictors and clinical features of postoperative hyperperfusion after surgical revascularization for moyamoya disease: a serial single photon emission CT/positron emission tomography study. Stroke. 2012;43(10):2610-2616.

29. Ladner TR, Donahue MJ, Arteaga DF, et al. Prior Infarcts, Reactivity, and Angiography in Moyamoya Disease (PIRAMD): a scoring system for moyamoya severity based on multimodal hemodynamic imaging. J Neurosurg. 2017;126(2):495-503.
30. Teo M, Furtado S, Kaneko OF, et al. Validation and application for the Berlin grading system of moyamoya disease in adult patients. Neurosurgery. 2020;86(2):203-212.

31. Sam K, Poublanc J, Sobczyk O, et al. Assessing the effect of unilateral cerebral revascularisation on the vascular reactivity of the non-intervened hemisphere: a retrospective observational study. BMJ Open. 2015;5(2):e006014.

\section{Disclosures}

The authors report no conflict of interest concerning the materials or methods used in this study or the findings specified in this paper.

\section{Author Contributions}

Conception and design: S Lee, Kim, W Park, JC Park, Kwon. Acquisition of data: all authors. Analysis and interpretation of data: S Lee, Kim, W Park, Ahn. Drafting the article: Kim, W Park. Critically revising the article: S Lee, Kim, W Park. Reviewed submitted version of manuscript: S Lee, Kim, W Park, Ahn. Approved the final version of the manuscript on behalf of all authors: S Lee. Statistical analysis: Kim. Administrative/ technical/material support: S Lee, Kim. Study supervision: S Lee, W Park, JC Park, Kwon, Ahn.

\section{Correspondence}

Seungjoo Lee: Asan Medical Center, University of Ulsan College of Medicine, Seoul, Republic of Korea. changhill@gmail.com. 